\title{
ERRATUM
}

Alberto Lovison • Franco Cardin • Alessia Bobbo

\section{Erratum to: Discrete structures equivalent to nonlinear Dirichlet and wave equations}

Published online: 16 April 2016

(C) Springer-Verlag Berlin Heidelberg 2016

Erratum to: Continuum Mech. Thermodyn. (2009) 21:27-40

DOI 10.1007/s00161-009-0097-1

\section{A perturbed wave equation amenable to exact reduction}

In [2] we have considered the problem of finding $T$-periodic solutions $u(t, x) \in H:=H_{0}^{1}\left([0, T] \times \mathbb{T}^{n}, \mathbb{R}\right)$ of the nonlinear wave equation

$$
\left\{\begin{array}{rl}
\square u(t, x) & =F(u), \\
u(t, x) & =u(t+T, x),
\end{array} \quad t \in \mathbb{R}, x \in \mathbb{T}^{n},\right.
$$

where $\square=\frac{\partial^{2}}{\partial t^{2}}-\frac{\partial^{2}}{\partial x^{2}}$ is the D'Alembert wave operator while $F$ is a Nemitski Lipschitz operator. To produce an exact finite reduction in the wave equation we needed the inverse operator of the D'Alembertian. To arrive to this scope we first expanded $v \in H$ in Fourier series and then we laid down the formal explicit component-wise inverse of $\square$ :

$$
\square^{-1}\left(v_{k}(t)\right):=\left(-\frac{1}{\sin (|k| T)} \int_{0}^{T} \sin (|k|(T-s)) v_{k}(s) \mathrm{d} s\right) \frac{\sin (|k| t)}{|k|}+\int_{0}^{t} \frac{\sin (|k|(t-s))}{|t|} v_{k}(s) \mathrm{d} s,
$$

where $k \in \mathbb{Z}^{n}, k \neq 0$.

However, this formula is not valid in general, i.e., the D'Alembert operator $\square$ does not admit a bounded inverse in $H$ both with rational and irrational periods $T$. This could be overcome by considering numbers of constant type

$$
|T k-l|>C k^{-1}
$$

as in [4]; this, however, is not compatible with the contractivity needed for the reduction procedure. We have instead adopted the standard approach by Rabinowitz [5], i.e., we considered the vibrating string $(n=1)$, we

The online version of the original article can be found under doi:10.1007/s00161-009-0097-1.

\footnotetext{
A. Lovison $(\varangle) \cdot$ F. Cardin $\cdot$ A. Bobbo

Dipartimento di Matematica Pura ed Applicata, Universitá degli Studi di Padova,

Via Trieste, 63, 35121 Padua, Italy

E-mail: lovison@math.unipd.it

F. Cardin

E-mail: cardin@math.unipd.it
} 
took $T$ rational multiple of $\pi$, and we restricted the domain of $\square$ to the orthogonal complement of the kernel of $\square$. The restricted problem appears in [1,3] among others. Adopting this format the kernel of the D'Alembertian becomes trivial and the existence of a bounded inverse $\square^{-1}$ is assured. In this way it is possible for instance to relax monotonicity conditions on $F$ and obtain existence results as well [3]. The procedure described in [2] is still valid and leads to the correct conclusions. A derivation of these results in the hypotheses discussed here has been exposed in full details in our subsequent work [6].

\section{References}

1. Brézis, H., Coron, J.-M., Nirenberg, L.: Free vibrations for a nonlinear wave equation and a theorem of P. Rabinowitz. Commun. Pure Appl. Math. 33, 667-684 (1980)

2. Lovison, A., Cardin, F., Bobbo, A.: Discrete structures equivalent to nonlinear Dirichlet and wave equations. Continuum Mech. Thermodyn. 21(1), 27-40 (2009)

3. Coron, J.-M.: Periodic solutions of a nonlinear wave equation without assumption of monotonicity. Math. Ann. 262, 273-285 (1983)

4. de la Llave, R.: Variational methods for quasi-periodic solutions of partial differential equations. In: Hamiltonian Systems and Celestial Mechanics (Pátzcuaro, 1998), Volume 6 of World Scientific Monograph Series in Mathematics, pp. 214-228. World Scientific Publishing, River Edge, NJ (2000)

5. Rabinowitz, P.H.: Periodic solutions of nonlinear hyperbolic partial differential equations. Commun. Pure Appl. Math. 20, 145-205 (1967)

6. Cardin, F., Lovison, A.: Finite mechanical proxies for a class of reducible continuum systems. Netw. Heterog. Media 9(3), 417-432 (2014) 